\title{
Cascade upconversion of photoluminescence in quantum dot ensembles
}

\author{
João R. Santos, ${ }^{1}$ Mikhail I. Vasilevskiy, ${ }^{1, *}$ and Sergey A. Filonovich ${ }^{2}$ \\ ${ }^{1}$ Centro de Física, Universidade do Minho, Campus de Gualtar, Braga 4710-057, Portugal \\ ${ }^{2}$ CENIMAT/I3N-CEMOP, Universidade Nova de Lisboa, Caparica 2829-516, Portugal
}

(Received 8 September 2008; published 22 December 2008)

\begin{abstract}
We present a theoretical model and Monte Carlo simulation results that naturally explain all the features of the thermally activated photoluminescence upconversion effect [also known as anti-Stokes photoluminescence (ASPL)] observed in ensembles of colloidal semiconductor nanocrystal quantum dots (QDs). The proposed ASPL mechanism includes the following principal ingredients: (i) optical-phonon-assisted absorption of an incident photon in a relatively large dot in the ensemble, (ii) emission of a higher-energy photon from the zero-phonon exciton-polaron state, with an upconversion equal to one optical-phonon energy, and (iii) cascade reabsorption and re-emission processes involving QDs of successively smaller sizes within the sample, rendering the experimentally observed large anti-Stokes shift of the energy of the photon that finally leaves the sample. The results obtained by the Monte Carlo modeling based on the proposed mechanism reproduce all the experimentally observed ASPL trends in colloidal QD solutions.
\end{abstract}

DOI: 10.1103/PhysRevB.78.245422

PACS number(s): 78.67.Bf

\section{INTRODUCTION}

Highly luminescent semiconductor nanocrystals (NCs) possess a number of interesting and important properties that are tunable, thanks to the size-dependent discrete electronic spectra characteristic of quantum dots (QDs). ${ }^{1}$ One of such properties is the upconverted (or anti-Stokes) photoluminescence (PL), i.e., the emission of photons with energy higher than that of the exciting photons. The anti-Stokes PL (ASPL) is of great interest for applications in multicolor displays, bioimaging systems, unconventional lasers, and solid-state optical refrigeration devices (see Ref. 2 and references therein). This effect is different from the second-harmonic generation, which is a coherent process resulting from nonlinear optical properties of the material. For the upconverted photoluminescence, carriers must be excited first into some intermediate states from where they can eventually proceed further to luminescent higher-energy states through one of the possible mechanisms such as absorption of another photon, Auger excitation, or energy accumulation from the phonon bath. The thermally activated upconversion effect in colloidal solutions of nearly spherical NCs obtained by wet chemistry methods was first observed in Ref. 3 . In contrast to the usual (Stokes-shifted) luminescence and also to the ASPL observed in other nanomaterials, such as porous silicon, ${ }^{4}$ polystyrene nanoparticles, ${ }^{5}$ and heterostructures with self-assembled QDs, ${ }^{6}$ the intensity of the upconverted emission of colloidal InP and CdSe QDs was found to increase with temperature ${ }^{3}$ implying the participation of phonons in this process. Since then, a number of studies of the upconversion effect in colloidal QDs have been published and the phonon-assisted ASPL has been reported for $\mathrm{CdSe},{ }^{7} \mathrm{CdTe},{ }^{8,9} \mathrm{PbS},{ }^{10}$ and PbSe (Ref. 11) NCs, as well as for $\mathrm{CdSe} / \mathrm{ZnS}$ core-shell nanoparticles. ${ }^{12,13}$

The thermally activated ASPL is observed when an ensemble of QDs is excited at the very edge of its absorption spectrum below the conventional PL band excited with highenergy photons. Figure 1 presents our experimental data ${ }^{14}$ showing the variation of the PL spectra with the change in the exciting photon energy $E_{\text {exc }}$ spanning a broad range from below the edge of the measurable absorption spectrum $\left(E_{\text {exc }}=2.03 \mathrm{eV}\right.$ and wavelength $\left.\lambda_{\text {exc }}=610 \mathrm{~nm}\right)$ to the absorption peak associated with the exciton recombination from its ground state (usually designated $\left.1 s_{e} 1 S_{3 / 2}\right)^{16}$ in QDs of the most probable size in the solution of $\mathrm{CdSe} / \mathrm{ZnS}$ nanocrystals. Beyond $\lambda_{\text {exc }}=550 \mathrm{~nm}$ the shape of the PL band practically does not change any more. It can be seen from Fig. 1 that, at a certain point (namely, near $\lambda_{\text {exc }}=575 \mathrm{~nm}$ ), the ASPL transforms into the usual Stokes-shifted PL. The gradual change in the band shape suggests that the physical processes involved in this emission are the same throughout the whole $E_{\text {exc }}$ range. A similar observation was reported recently for $\mathrm{PbS}$ QDs. ${ }^{10}$

The principal experimental facts concerning the upconversion effect in nanocrystal ensembles can be summarized as follows: ${ }^{2}$

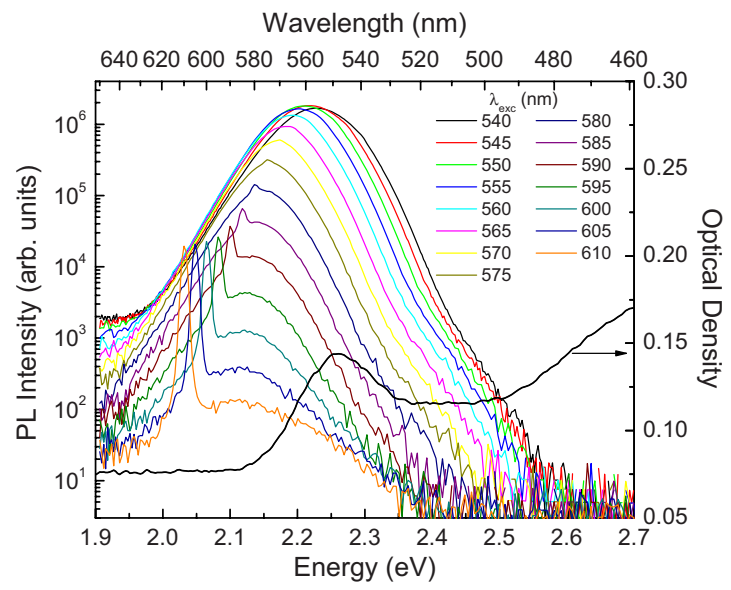

FIG. 1. (Color online) Room-temperature (RT) PL spectra of a colloidal solution of $\mathrm{CdSe} / \mathrm{ZnS}$ core-shell crystalline nanoparticles (core diameter: $4 \mathrm{~nm}$; shell thickness: $0.5 \mathrm{~nm}$; Refs. 14 and 15). The spectra were excited with light of different wavelengths as indicated on the plot and measured in the perpendicular collection mode. Also shown is the absorption spectrum of the solution. 
(i) The ASPL intensity increases linearly with the excitation power, which can be rather low. It also increases strongly with temperature.

(ii) The shift between the ASPL peak position and $E_{\text {exc }}$ does not significantly depend on the QD size if $E_{\text {exc }}$ is chosen proportionally to the absorption peak energy (which is, of course, size dependent). However, the shift between the maximum of the upconverted band and $E_{\text {exc }}$ increases with the temperature and also with the optical thickness of the sample (see below). It can range approximately from 20 to $150 \mathrm{meV}$.

(iii) The ASPL band becomes broader for lower $E_{\text {exc }}$ and the upconversion energy can reach more than $400 \mathrm{meV}$ at the edge of the band.

Possible ASPL mechanisms were discussed in Refs. 3, 7, and 8 , but no convincing conclusion was made. By virtue of (i), processes such as two-photon absorption and Auger excitation can be excluded in this case. It looks most plausible that incident photons excite electrons to some intermediate states from which they eventually proceed to the higherenergy (luminescent) states, which are the same as in the case of usual PL, through a thermal activation effect. It was suggested $^{3,7,8}$ that the intermediate (gap) states are localized at the NC surface, i.e., are not intrinsic to the QD. However, this interpretation is hardly acceptable. According to (ii), the surface states' energy then should scale with the NC size in the same way as does the exciton ground-state energy (roughly as $R^{-2}$, where $R$ is the QD radius), which is not possible from the theoretical point of view. ${ }^{14}$ Also, the fact that the ASPL efficiency increases with the quantum yield of the QDs (Ref. 13) provides another strong argument against the interpretation of the intermediate states as surface ones. Since the ASPL has been observed for several different types of NCs, the intermediate states involved in the mechanism must be intrinsic to QDs, independently of their material and covering shell. Thus, no satisfactory explanation of the physics involved in the ASPL phenomenon has been given so far.

In this work we present our model of the thermally activated ASPL based on the QD polaron concept and including (i) phonon-assisted upconversion in an individual dot and (ii) cascade reabsorption and re-emission processes involving QDs of successively smaller sizes within the sample. We also provide additional experimental data (Figs. 1 and 2) confirming the conclusions of our modeling. The paper is organized as follows. In Sec. II we describe the model and the Monte Carlo (MC) simulation procedure based on it is explained in Sec. III. The calculated results are presented and discussed in Sec. IV which is ended by conclusions.

\section{MODEL}

It is known that the energy spectrum of electron-hole pairs (conventionally called excitons) in QDs is strongly modified due to the interaction with optical phonons that is enhanced compared to bulk semiconductors because of the electron, hole, and phonon confinement. ${ }^{17}$ The true stationary states of the interacting system consisting of an exciton and phonons are called exciton-polaron states, ${ }^{18}$ which have been observed experimentally in nanocrystal QDs by using size-

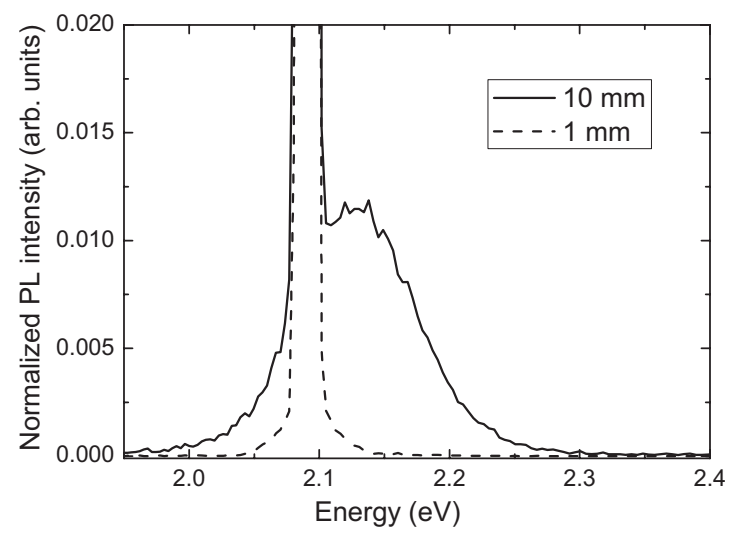

FIG. 2. RT emission spectra of the same colloidal solution of $\mathrm{CdSe} / \mathrm{ZnS}$ core-shell nanoparticles (Refs. 14 and 15) placed into two cuvettes of different thicknesses $(1$ and $10 \mathrm{~mm})$. The measurements were performed in the perpendicular collection mode using the excitation at $590 \mathrm{~nm}$.

selective PL (Ref. 19) and pump-and-probe transmission ${ }^{20}$ spectroscopy. In the simplest case of just one "bare" exciton level $\left(E_{0}\right)$ and one optical-phonon mode (of energy $\left.\hbar \omega_{0}\right)$, the so-called "independent boson model," 21 the polaron spectrum consists of a series of equidistant levels separated by $\hbar \omega_{0}$, with the ground state [called zero-phonon line (ZPL)] located at $E_{0}-\Delta$, where $\Delta$ depends on the exciton-phonon coupling strength. The corresponding absorption and emission spectra consist of series of equidistant $\delta$ peaks of unequal intensity and they are the mirror image of each other with respect to ZPL. Within the independent boson model, the absorption $\left(\sigma_{a}\right)$ and emission $\left(\sigma_{e}\right)$ cross sections of an individual QD are determined by the spectral function, ${ }^{21}$

$$
\begin{aligned}
& A(E)= 2 \pi \exp \{-S[2 \bar{n}(T)+1]\} \\
& \times \sum_{n=-\infty}^{+\infty}\left[\frac{\bar{n}(T)+1}{\bar{n}(T)}\right]^{n / 2} I_{n}\{2 S \sqrt{\bar{n}(T)[\bar{n}(T)+1]}\} \\
& \times \delta\left(E-E_{0}+\Delta-n \hbar \omega_{0}\right), \\
& \sigma_{a}(E) \propto A(E), \quad \sigma_{e}(E) \propto \exp [-E / k T] A(E),
\end{aligned}
$$

where $S$ is the Huang-Rhys parameter, $\bar{n}(T)$ is the mean number of the optical phonons at temperature $T$ (given by the Bose-Einstein function), $I_{n}$ denotes the modified Bessel function, and $\Delta \approx S \hbar \omega_{0} .{ }^{22}$ Taking into account the polaron effect has allowed for the explanation of several peculiarities in the PL (Refs. 23-25) and multiphonon $\operatorname{Raman}^{26}$ spectra of QDs.

It has been suggested ${ }^{23,27}$ that the polaron absorption peaks lying beneath the exciton ground energy level (e.g., the "-1LO" satellite; see Fig. 3) can be responsible for the excitation of QDs below the interband absorption limit. After absorbing a photon and reaching thermal equilibrium, the excited dot can emit a photon most likely with the energy corresponding to the ZPL or (more rarely) with that corresponding to the "1LO" satellite in the emission spectrum. The energy gain of $\hbar \omega_{0}$ or $2 \hbar \omega_{0}$, respectively, comes from the thermostat that maintains the dot at a given temperature. This explains why the anti-Stokes shift (i.e., the gap between 

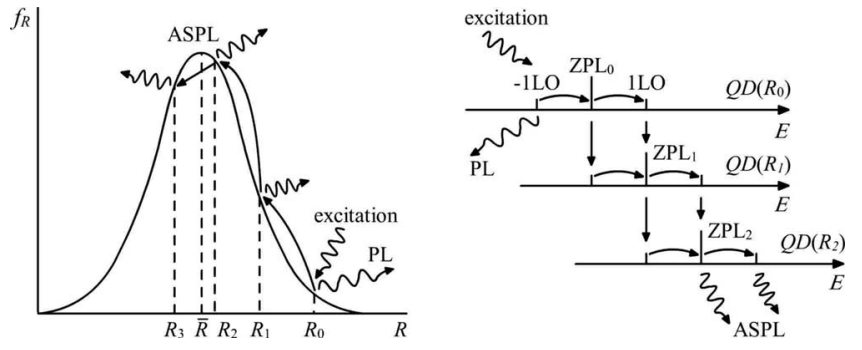

FIG. 3. Cascade energy-transfer process in an ensemble of QDs with the radius distribution shown in the left panel. A large dot, of radius $R_{0}$, is excited through its $-1 \mathrm{LO}$ absorption peak and emits through either ZPL or " $1 \mathrm{LO}$ " satellite, with an upconversion equal to $\hbar \omega_{0}$ or $2 \hbar \omega_{0}$, respectively. The emitted photon eventually is reabsorbed by a smaller dot, of radius $R_{1}$, and then re-emitted, in some cases with a higher energy. At each step, some photons leave the sample while some are reabsorbed by still smaller QDs. The process repeats until the number of dots able to reabsorb the photon becomes too small.

the absorbing and emitting states) practically does not depend on the QD radius, because the energy of confined optical phonons changes only very weakly with $R .^{28}$ The strongly increasing temperature dependence of the ASPL intensity is naturally explained by the raising of the satellite peaks in both absorption and emission. ${ }^{2,23}$ However, it seems to be difficult to explain the observed values of the upconverted shift which are much larger than the LO phonon energy. ${ }^{2}$ Of course, there are " $-n$ LO" and " $n$ LO" satellites with $n>1$ in the QD spectra, but their intensities are much too low for realistic values of the electron-phonon coupling constants. ${ }^{26}$

A clue to the understanding of the large anti-Stokes shift is the experimentally established fact that the ASPL can occur only in samples with a sufficiently long optical pathway. We observed this effect by measuring PL spectra of two samples with different optical thicknesses, which are otherwise completely equivalent. The same solution of $\mathrm{CdSe} / \mathrm{ZnS}$ core-shell nanoparticles was placed into two cuvettes of different thicknesses and the PL spectra of these two samples are presented in Fig. 2. If the optical density of the sample is high enough, reabsorption and re-emission of photons by different QDs within it are possible and the spectral shift larger than $\hbar \omega_{0}$ can be explained by a cascade excitation process as schematically shown in Fig. 3 (where the absorption and emission spectra of individual dots are taken within the independent boson model for simplicity). Note that the situation considered here differs from the majority of the cases in which incoherent radiation transport is important since there is a distribution of species' sizes (and therefore the spectra), contrary to the most common case in which all the species are spectrally equivalent. ${ }^{29}$

\section{MONTE CARLO MODELING PROCEDURE}

We performed a MC modeling of the processes sketched in Fig. 3 based on the polaron theory for the probabilities of the elementary absorption and emission events and considering only the $n=0$ (ZPL) and $n= \pm 1$ terms in Eq. (1). Sup- pose that at $t=0$ there is one excited QD of a certain radius $R_{0}$ whose $1 \mathrm{LO}$ satellite peak is resonant to the exciting photon energy $\left(E_{\mathrm{exc}}=E_{0}-\Delta-\hbar \omega_{0}\right)$ and this dot is located somewhere in the middle of the sample. ${ }^{30}$ Within a short time (of the order of the characteristic time of the QD exciton radiative recombination), this dot will emit a photon of one of the following three energies: $E_{0}-\Delta-\hbar \omega_{0}, E_{0}-\Delta$, or $E_{0}-\Delta$ $+\hbar \omega_{0}$. The relative probabilities of the three possible events are proportional to the corresponding values of $\sigma_{e}$ given by Eq. (1). Once the first photon has been emitted according to this probability distribution, its destiny can be one of the following: (i) it simply leaves the sample and (as we assume) is registered by the detector or (ii) it is reabsorbed by another QD of appropriate size. In the latter case, there are three possibilities (corresponding to three different QDs), namely, of the photon being absorbed through the ZPL of the secondary dot or through one of its $n= \pm 1$ satellites. If one of the reabsorption events considered in Fig. 3 takes place, the excited QD (that can have the radius $R_{0}, R_{1}$, or eventually $R_{2}$; see Fig. 3) will emit a secondary photon (for which now there are five possible values of the energy) whose destiny is determined by the same rules and the cascade process continues like this. Then, after a certain number of reabsorptions the photon definitely leaves the sample because of its random-walk motion mediated by elastic-scattering events (occurring much more frequently than the reabsorption and re-emission ones).

Our description of the stochastic radiative transport in the solution of absorbing and emitting nanoparticles is based on the master-equation formalism (see the Appendix). Because of the factorization of space and time dependences of the photon populations inside the sample, ${ }^{31}$ the simulation procedure can be restricted to the temporal aspects of the radiative transport, while the finite size of the sample is taken into account indirectly as explained below. The MC method is used to reproduce the evolution of photons within the defined volume. Each MC cycle begins with the creation of one photon as explained above and ends by the detection of one emitted photon. Within the spirit of the MC modeling, we choose an observation time $\Delta t$ as a typical time that the emitted photon would need to reach the sample's surface and be detected (unless it is reabsorbed). Assuming that the photon's propagation inside the solution is diffusive due to nonresonant Rayleigh scattering events, the observation time can be estimated as $\Delta t \approx L^{2} / D$, where $L$ is a characteristic size of the sample and $D$ is the diffusion coefficient. Then, using Eq. (1), the (relatively low) probability of the reabsorption of a photon (of energy $E_{1}$ ) can be calculated as

$$
\begin{aligned}
P_{1}= & N(c / \eta)\left[\sigma_{a}^{(-1)} f\left(E_{1}+\hbar \omega_{0}\right)+\sigma_{a}^{(0)} f\left(E_{1}\right)\right. \\
& \left.+\sigma_{a}^{(1)} f\left(E_{1}-\hbar \omega_{0}\right)\right] \Delta t
\end{aligned}
$$

where $N$ is the overall QD concentration, $c$ is the velocity of light, $\eta$ is the refraction index of the medium where the NCs are embedded, $\sigma_{a}^{(j)}$ is the absorption cross section of the $j \mathrm{LO}$ satellite peak, and $f$ denotes the distribution function of the bare exciton energies in the dot. The latter is related to the QD radius distribution function $f_{R}$ shown schematically in Fig. 3 and normalized by the condition $\Sigma_{R} f_{R}=1$ according to 


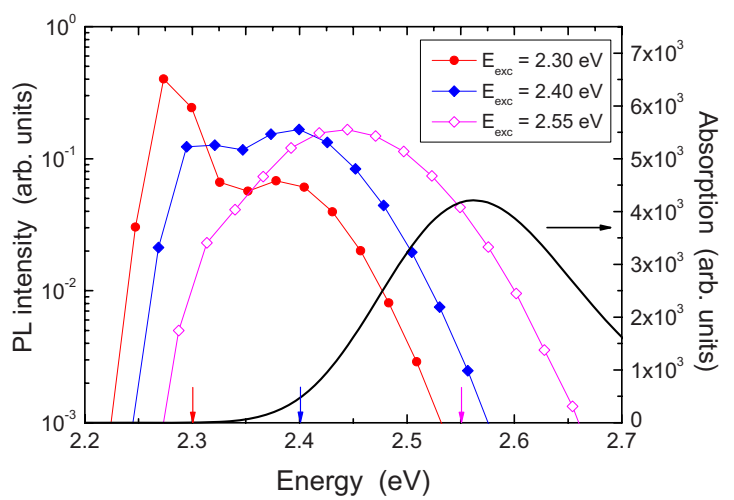

FIG. 4. (Color online) Modeled RT emission and absorption spectra of a colloidal solution of CdSe QDs. The emission spectra were calculated for three different values of the incident photon energy using the Monte Carlo procedure. The QD radius distribution function $f_{R}$ was taken as a Gaussian with $\bar{R}=2 \mathrm{~nm}$ (corresponding to $\bar{E}=2.56 \mathrm{eV}$ ) and standard deviation of $5 \%$. The values of $\hbar \omega_{0}=26.2 \mathrm{meV}$ for the optical-phonon energy and $S=0.2$ for the Huang-Rhys parameter were used. The absorption spectrum was calculated using the effective-mass approximation as explained in Ref. 32.

$f_{R}=f(E(R))$, where $E(R)$ is given by the known relation obtained within the effective-mass approximation. ${ }^{16,28,32}$ Random numbers were used at each elementary step in order to decide, (i) what is the energy $\left(E_{1}\right)$ of the photon emitted by the excited QD and (ii) which one of the three possibilities of the reabsorption considered in Eq. (2) takes place or the photon just leaves the sample. If the latter is not the case, another emission and reabsorption/escape step takes place according to the same rules. However, one change must be made in Eq. (2) in order to describe the steps number $i>1$. The successive reabsorption events take place, in average, each time closer to the sample's surface and, consequently, the observation time shrinks with the increase in $i$. According to the random-walk theory, we can expect that $\Delta t^{(i)}=\Delta t[1-C(i$ $-1)$ ] where $C$ is a constant depending on the sample size and QD concentration (see the Appendix). The necessary replacements in Eq. (2) for $P_{i}$ (with $i>1$ ) are $E_{1} \rightarrow E_{i}$ and $\Delta t$ $\rightarrow \Delta t^{(i)}$. Each calculated PL spectrum was obtained by averaging over some $10^{6} \mathrm{MC}$ cycles.

\section{RESULTS AND DISCUSSION}

Some results of our MC modeling performed for CdSe QDs are presented in Figs. 4-6. As it can be seen from Figs. 4 and 5, the upconversion effect indeed takes place only when the impinging photons' energy is below the interband absorption limit for most of the dots. When $E_{\text {exc }}$ falls into the spectral region corresponding to the ZPL absorption in many QDs in the sample, the cascade process of reabsorption/reemission events goes most likely in the opposite direction, i.e., from smaller to larger dots. This, of course, leads to the (usual) Stokes-shifted PL (Fig. 4, $E_{\text {exc }}=2.55 \mathrm{eV}$ ). The upconversion process is still possible but it is rare and unimportant in this situation because of the lack of smaller dots. The transition between the two regimes (ASPL and conven-

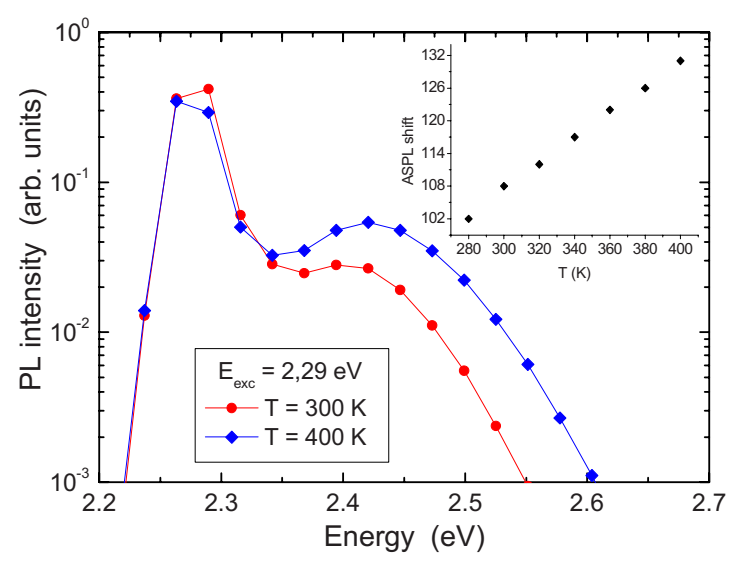

FIG. 5. (Color online) PL spectra of the colloidal solution of CdSe QDs of Fig. 3 calculated for two different temperatures. The inset shows the variation of the peak position of the ASPL band with temperature.

tional PL) takes place gradually with the increase in $E_{\text {exc }}$, in agreement with our experimental data presented in Fig. 1.

According to Fig. 5, the increase in temperature leads to a more intense and also more blueshifted ASPL band. Both effects are due to the higher magnitude of the $n= \pm 1$ satellites in the QD polaron spectrum. Because of this, the probability of upconversion in individual dots is higher and the average number of steps in the cascade process $(\bar{i})$ also increases. While the shift of the maximum of the ASPL band (determined by $\bar{i}$ ) increases approximately linearly with temperature (see inset of Fig. 5), the peak intensity grows superlinearly, in accordance with experiment. ${ }^{2}$ Finally, the modeling results also confirm our experimental observation that the ASPL is possible only when the optical path inside the sample is long enough. As demonstrated by Fig. 6, the ASPL develops only if there is a sufficiently large number of reabsorption and re-emission events; otherwise, the PL spectrum is only broadened. This is in complete agreement with the experimental results shown in Fig. 2.

It should be pointed out that the phenomenon of luminescence self-absorption is well known in the literature. ${ }^{33}$ In particular, the observed emission band can become darker in the center than on the sides because of the reabsorption in an ensemble of spectrally identical species, the so-called "line
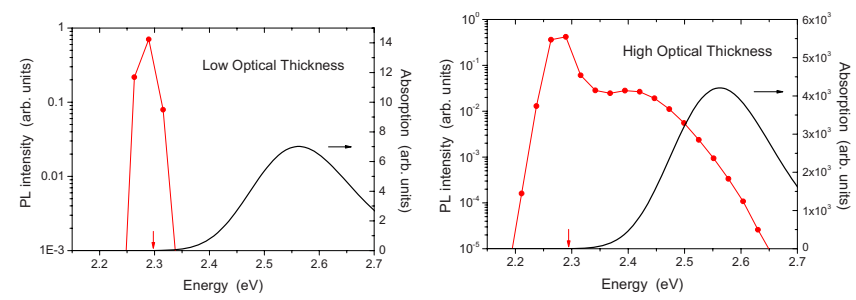

FIG. 6. (Color online) Calculated RT absorption and emission spectra of two NC solutions with different optical densities. The spectra presented in the two panels were calculated assuming two different values of the observation time $\Delta t$ that mimics the different optical thicknesses of the two samples (while all the other parameters are kept the same). The red vertical arrows indicate the excitation energy. 
self-reversal" effect, which is known both experimentally and theoretically ${ }^{33}$ and also has been modeled numerically. ${ }^{34}$ However, in our case where the bare QD spectrum consists of separate narrow lines (ZPL and its satellites), it is essential to have a (pseudo)continuous distribution of emitting/ absorbing species in order to explain the experimentally observed PL band, which can be redshifted or blueshifted depending on the excitation wavelength.

In conclusion, we have shown that the present model taking into account the polaron-mediated upconversion in an individual QD and the radiative energy transfer from larger dots to more numerous smaller ones explains all the principal features of the phonon-assisted ASPL in colloidal solutions. It indicates that the ASPL effect is enhanced when the optical density of the sample (i.e., the QD concentration) is higher and the exciton-phonon interaction in the dots is stronger, e.g., for core-shell nanoparticles where the spatial separation of the electron and hole wave functions leads to an increased exciton coupling to optical phonons through the Fröhlich mechanism. We believe that the proposed cascade upconversion mechanism is not limited to nanocrystal colloidal solutions but can also take place in other systems such as, e.g., QDs coated onto a polystyrene microsphere. ${ }^{35}$ The understanding of the ASPL mechanism thus opens the possibility of tailoring the parameters of nanocrystal QD ensembles for a manifold of photonic applications.

\section{ACKNOWLEDGMENTS}

This work was supported by the Portuguese Foundation for Science and Technology (FCT). The authors wish to thank D. V. Talapin and A. L. Rogach for their sample preparation and characterization and E. Pereira and Yu. P. Rakovich for useful discussions.

\section{APPENDIX}

First, we derive Eq. (2) of the paper. Let us assume that an excited QD emits a photon of energy $E_{0}$ at $t=0$. The probability of survival $p(t)$ of this photon inside the medium without being absorbed until a moment of time $t$ is described by the master equation

$$
\frac{d p(t)}{d t}=-p(t) \frac{c}{\eta} N \sum_{j=-1}^{1} \sigma_{a}^{(j)} f\left(E_{0}-j \hbar \omega_{0}\right),
$$

where $N$ is the total QD concentration. By integrating Eq. (A1) we obtain the reabsorption probability

$$
P=1-p(t)=1-\exp \left\{-\left[\frac{c}{\eta} N \sum_{j=-1}^{1} \sigma_{a}^{(j)} f\left(E_{0}-j \hbar \omega_{0}\right)\right] t\right\} .
$$

If the observation time introduced in the text $(\Delta t)$ is sufficiently small, Eq. (A2) reduces to Eq. (2), i.e., the absorption probability is proportional to the time.

The MC observation time is reduced with the number of reabsorption events. In order to demonstrate this, let us assume that the sample has a spherical shape (of radius $L$ ). The primary QD is located in its center and the detectors are placed everywhere at the sample surface. We also assume that the photon transport can approximately be described by the diffusion equation since the random walk of photons inside the sample occurs mostly because of their elastic nonresonant scattering. The processes of resonant absorption and re-emission of photons are relatively seldom and the distribution of excited QDs in space and time can be approximated by the spherical-symmetric solution of the diffusion equation

$$
n(\mathbf{r}, t)=\frac{A}{(D t)^{3 / 2}} \exp \left(-\frac{r^{2}}{4 D t}\right),
$$

where $D$ is the diffusion coefficient and the normalization constant is chosen to obey the condition $4 \pi \int_{0}^{L} n(\mathbf{r}, t) r^{2} d r=1$. After a time $t$, the excited dot will be located, on average, at a distance $\langle r(t)\rangle \propto \sqrt{D t}$ from the center. Its distance from the detector (that is, from the surface) averaged over all possible directions of the photon emission will be equal to $l=L$ $-\langle r(t)\rangle^{2} / 3 L$. Calculating the mean value with the help of Eq. (A3) we obtain (for $L \rightarrow \infty$ )

$$
l=L\left(1-2 D t / L^{2}\right) .
$$

During this time, the photon has participated in some $(i-1)$ absorption/re-emission events $(i=1,2, \ldots)$, so that we have $t=\tau(i-1)$, where $\tau$ is the inverse of the absorption probability per unit time, discussed in the text. Then we can admit that the observation time for the $i$ th step, equal to the time necessary for the photon to reach the surface with respect to the moment $t$, will decrease with $t$ as $\Delta t(t) \approx l^{2} / D=\Delta t(0)[1$ $-2 t / \Delta t(0)]$, where $\Delta t(0)=L^{2} / D$ was denoted simply as $\Delta t$ in the text. Thus,

$$
\Delta t^{(i)}=\Delta t[1-C(i-1)],
$$

where $C=2\left[\frac{L^{2} N c}{D \eta} \sum_{j=-1}^{1} \sigma_{a}^{(j)} f\left(E_{0}-j \hbar \omega_{0}\right)\right]^{-1} \approx 2\left(P_{1}\right)^{-1}$ with $P_{1}$ defined by Eq. (2).

\footnotetext{
*Corresponding author; mikhail@ fisica.uminho.pt

${ }^{1}$ A. P. Alivisatos, Science 271, 933 (1996).

${ }^{2}$ Yu. P. Rakovich and J. F. Donegan, in Semiconductor Nanocrystal Quantum Dots. Synthesis, Assembly, Spectroscopy and Applications, edited by A. L. Rogach (Springer, Wien, NY, 2008), pp. 257-275.
}

${ }^{3}$ E. Poles, D. C. Selmarten, O. I. Mićić, and A. J. Nozik, Appl. Phys. Lett. 75, 971 (1999).

${ }^{4}$ D. Kovalev, J. Diener, H. Heckler, G. Polisski, N. Künzner, F. Koch, Al. L. Efros, and M. Rosen, Phys. Rev. B 61, 15841 (2000).

${ }^{5}$ K. Dou, Z. Xu, X. J. Wang, A. Apblett, and T. Collins, J. Lumin. 
108, 355 (2004).

${ }^{6}$ M. Y. Valakh, N. O. Korsunska, Yu. G. Sadofyev, V. V. Strelchuk, G. N. Semenova, L. V. Barkovska, V. V. Artamonov, and M. V. Vuychik, Mater. Sci. Eng., B 101, 255 (2003).

${ }^{7}$ Y. P. Rakovich, S. A. Filonovich, M. J. M. Gomes, J. F. Donegan, D. V. Talapin, A. L. Rogach, and A. Eychmüller, Phys. Status Solidi B 229, 449 (2002).

${ }^{8}$ X. Wang, W. W. Yu, J. Zhang, J. Aldana, X. Peng, and M. Xiao, Phys. Rev. B 68, 125318 (2003).

${ }^{9}$ Y. P. Rakovich, A. A. Gladyshchuk, K. I. Rusakov, S. A. Filonovich, M. J. M. Gomes, D. V. Talapin, A. L. Rogach, and A. Eychmüller, J. Appl. Spectrosc. 69, 444 (2002).

${ }^{10}$ M. J. Fernée, P. Jensen, and H. Rubinsztein-Dunlop, Appl. Phys. Lett. 91, 043112 (2007).

${ }^{11}$ J. M. Harbold and F. W. Wise, Phys. Rev. B 76, 125304 (2007).

${ }^{12}$ K. I. Rusakov, A. A. Gladyshchuk, Y. P. Rakovich, J. F. Donegan, S. A. Filonovich, M. J. M. Gomes, D. V. Talapin, A. L. Rogach, and A. Eychmüller, Opt. Spectrosc. 94, 859 (2003).

${ }^{13}$ Y. P. Rakovich, J. F. Donegan, S. A. Filonovich, M. J. M. Gomes, D. V. Talapin, A. L. Rogach, and A. Eychmüller, Physica E 17, 99 (2003).

${ }^{14}$ Al. L. Efros, M. Rosen, M. Kuno, M. Nirmal, D. J. Norris, and M. Bawendi, Phys. Rev. B 54, 4843 (1996).

${ }^{15} \mathrm{CdSe} / \mathrm{ZnSe}$ core-shell nanoparticles used in this work were prepared by an organometallic synthetic approach in a threecomponent hexadecylamine-trioctylphosphine oxidetrioctylphosphine mixture as described in Ref. 16. For the PL measurements, the nanocrystals were dissolved in toluene. The PL spectra were excited using a Xe lamp and a monochromator and measured with a Hamamatsu R943 photomultiplier.

${ }^{16}$ D. V. Talapin, A. L. Rogach, A. Kornowski, M. Haase, and H. Weller, Nano Lett. 1, 207 (2001).

${ }^{17}$ M. I. Vasilevskiy, in Semiconductor Nanocrystal Quantum Dots. Synthesis, Assembly, Spectroscopy and Applications, edited by A. L. Rogach (Springer, Wien, NY, 2008), pp. 217-255.

${ }^{18}$ O. Verzelen, R. Ferreira, and G. Bastard, Phys. Rev. Lett. 88, 146803 (2002).
${ }^{19}$ T. Itoh, M. Nishijima, A. I. Ekimov, C. Gourdon, Al. L. Efros, and M. Rosen, Phys. Rev. Lett. 74, 1645 (1995).

${ }^{20}$ J. Valenta, J. Moniatte, P. Gilliot, B. Hönerlage, J. B. Grun, R. Levy, and A. I. Ekimov, Phys. Rev. B 57, 1774 (1998).

${ }^{21}$ G. D. Mahan, Many-Particle Physics (Plenum, New York, 1990).

${ }^{22}$ Absorption and emission spectra of QDs beyond the independent boson model were calculated in Ref. 23.

${ }^{23}$ M. I. Vasilevskiy, E. V. Anda, and S. S. Makler, Phys. Rev. B 70, 035318 (2004).

${ }^{24}$ V. M. Fomin, V. N. Gladilin, J. T. Devreese, E. P. Pokatilov, S. N. Balaban, and S. N. Klimin, Phys. Rev. B 57, 2415 (1998).

${ }^{25}$ T. J. Liptay, L. F. Marshall, P. S. Rao, R. J. Ram, and M. G. Bawendi, Phys. Rev. B 76, 155314 (2007).

${ }^{26}$ R. P. Miranda, M. I. Vasilevskiy, and C. Trallero-Giner, Phys. Rev. B 74, 115317 (2006).

${ }^{27}$ K. Král, P. Zděnek, and Z. Khás, Surf. Sci. 566-568, 321 (2004).

${ }^{28}$ A. G. Rolo and M. I. Vasilevskiy, J. Raman Spectrosc. 38, 618 (2007).

${ }^{29}$ Dynamics of Radiation in Resonance Energy Transfer, edited by D. L. Andrews and A. A. Demidov (Wiley, Chichester, 1999).

${ }^{30}$ Owing to the high optical density of the medium, the absorption of the exciting beam and, consequently, the probability of excitation of the "primary" dots are not homogeneous in space. However, we do not take this fact into account because it would increase drastically the mathematical complexity of the problem, without influencing the qualitative understanding of the effect.

${ }^{31}$ E. Nunes Pereira, M. N. Berberan-Santos, and J. M. G. Martinho, J. Chem. Phys. 104, 8950 (1996).

${ }^{32}$ S. A. Filonovich, Y. P. Rakovitch, M. I. Vasilevskiy, M. V. Artemyev, D. V. Talapin, A. L. Rogach, A. G. Rolo, and M. J. M. Gomes, Monatsch. Chem. 133, 909 (2002).

${ }^{33}$ R. D. Cowan and G. H. Dieke, Rev. Mod. Phys. 20, 418 (1948).

${ }^{34}$ A. R. Alves-Pereira, E. J. Nunes-Pereira, J. M. G. Martinho, and M. N. Berberan-Santos, Eur. J. Phys. 28, 1105 (2007).

${ }^{35}$ N. Gaponik, Y. P. Rakovich, M. Gerlach, J. F. Donegan, D. Savateeva, and A. L. Rogach, Nanoscale Res. Lett. 1, 68 (2006). 